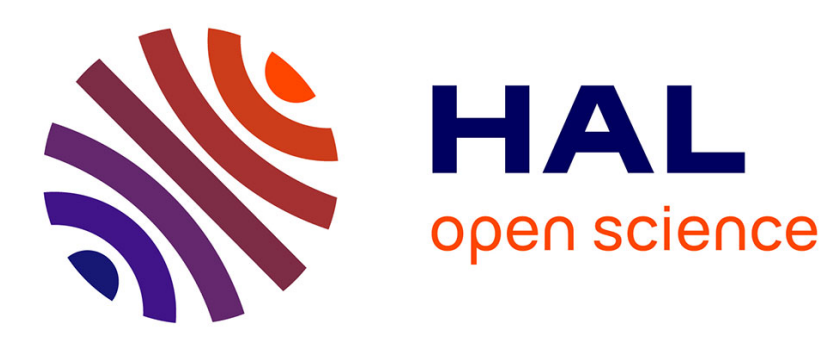

\title{
Dire quelque chose de soi : la théorie du genre de Judith Butler comme autofiction philosophique
}

\author{
Nathanaël Wadbled
}

\section{To cite this version:}

Nathanaël Wadbled. Dire quelque chose de soi : la théorie du genre de Judith Butler comme autofiction philosophique. Arnaud Genon; Isabelle Grell. Lisières de l'autofiction. Enjeux géographiques, artistiques et politiques, Presses universitaires de Lyon (PUL), pp.259-276, 2016, 978-2-7297-0899-3. hal-01654363

\section{HAL Id: hal-01654363 https://hal.science/hal-01654363}

Submitted on 3 Dec 2017

HAL is a multi-disciplinary open access archive for the deposit and dissemination of scientific research documents, whether they are published or not. The documents may come from teaching and research institutions in France or abroad, or from public or private research centers.
L'archive ouverte pluridisciplinaire HAL, est destinée au dépôt et à la diffusion de documents scientifiques de niveau recherche, publiés ou non, émanant des établissements d'enseignement et de recherche français ou étrangers, des laboratoires publics ou privés. 


\section{Nathanaël Wadbled \\ Dire quelque chose de soi :}

\section{la théorie du genre de Judith Butler comme autofiction philosophique}

In Arnaud Genon et Isabelle Grell (dirs.), Lisières de l'autofiction. Enjeux géographiques, artistiques et politiques, Lyon, PUL, 2016, p. 259-276.

\section{UNE PRATIQUE PHILOSOPHIQUE FICTIONNELLE ET REFERENTIELLE}

Les ouvrages philosophiques de Judith Butler semblent prendre une forme fictionnelle et autoréférentielle de par la posture de leur auteure. Si Butler revendique écrire de la philosophie, elle ne saurait se résumer à cela. Elle revendique publier également comme féministe et est reconnue comme telle. Butler offre la représentation d'une situation sociale ne cherchant pas à donner une définition ontologique, mais à comprendre la manière dont ces identités se produisent et se jouent. Cette démarche semble se situer à la convergence de deux articulations : réalité-fiction et philosophie-récit fictionnel. Il ne s'agit ni de décrire la réalité en apportant une garantie méthodologique à ce qui en est dit - comme la sociologie, l'histoire mais aussi la biographie -, ni de décrire un pur monde de langage et de faire de la métaphysique. La déconstruction de la réalité la produit comme fiction dont il s'agit de rendre compte.

Le résultat d'un tel travail est une fiction, par opposition à la description d'une réalité tangible et vérifiable, et par opposition à une théorie métaphysique. S'inscrivant comme héritière du mouvement féministe, Butler l'est donc d'une action revendicatrice par laquelle des individus minoritaires prennent la parole pour affirmer leur existence. C'est en tant que femme et que lesbienne qu'elle écrit une théorie de la formation de l'identité des femmes et des lesbiennes. La difficulté se trouve donc au niveau de la reconnaissance de cette référentialité, c'est-à-dire au niveau du pacte de lecture. Tant qu'il est devant un traité de philosophie, le lecteur situe ce qu'il lit comme ne renvoyant pas une expérience concrète réelle. En même temps, en tant que lecteur d'un ouvrage féministe activiste, il le situe comme renvoyant à l'expérience de son auteure. 
Au-delà des définitions plus ou moins discriminantes données à l'autofiction, cette fictionnalisation et cette auto-référentialité inhérentes à la position et à l'attitude de Butler peuvent faire légitimement se poser la question du caractère autofictionnel de cette philosophie. Semble mis en place un pacte autobiographique et un pacte philosophique : un auteur informe sans ambiguïté qu'il renvoie à une histoire vraie tout en se plaçant explicitement sur un plan théorique général. La fiction s'assume comme une philosophie référentielle. En tant qu'œuvre de philosophie, elle suit l'exigence doubrovskienne selon laquelle une autofiction doit se présenter comme un roman. En déconstruisant son expérience de femme et de lesbienne, elle assume explicitement une autoréférentialité. Si elle n'utilise pas la première personne, suivant la norme de l'écriture philosophique académique, l'identité nominale entre l'auteure, le narrateur et le personnage semble être clairement assumée et revendiquée.

Butler semble, par son style, s'adonner à une certaine abstraction, mais son écriture s'attache cependant à décrire des processus concrets dont elle agence les éléments. Elle pratique une déconstruction généalogique, ce qui suppose la description de processus et d'évolutions chronologiquement déterminés. Cette généalogie n'a pas une forme explicitement historique ou anthropologique. Elle fait de la philosophie d'une manière apparemment plus classique que Michel Foucault, dans la mesure où elle ne fait pas référence à des individus nommément identifiables, ni à des événements clairement spécifiés et datés. Son écriture se donne sous le mode de mises en intrigue articulant et synthétisant ce qu'elle considère comme les moments de processus de subjectivation genrée. Elle raconte dans un récit la constitution du genre : l'interpellation d'Althusser, la constitution du sujet mélancolique, la dialectique du maître et du valet ${ }^{1}$, etc. Plus son travail est théorique, plus elle recourt à la forme du récit, comme pour compenser l'absence de récit descriptif présent. Cela apparaît bien dans le dernier chapitre du Pouvoir des mots où elle fait jouer et dialoguer les conceptions bourdieusienne et derridienne de la performativité, en composant une intrigue passant d'une focalisation à l'autre et produisant une tension narrative $\mathrm{e}^{2}$. Tout travail théorique n'est pas pour autant un récit, dans la mesure où ils ne sont pas tous composés sous forme d'intrigue. Ces éléments théoriques, aussi bien que ceux des récits vraisemblables, n'existent que dans le réseau d'interactions déterminées par une progression quasi chronologique, et non

\footnotetext{
${ }^{1}$ En particulier dans Judith Butler, La Vie psychique du pouvoir : l'assujettissement en théories [1997], Brice Matthieussent (trad.), Paris, Léo Scheer, 2002. Cet ouvrage est le plus théorique et conceptuel.

2 Judith Butler, «Censure implicite et puissance d'agir discursive», dans Le Pouvoir des mots : politique du performatif [1997], Charlotte Nordmann \& Jérôme Vidal (trad.), Paris, Éditions Amsterdam, 2004.
} 
seulement par des structures logiques. Elle raconte des histoires conceptuelles, des récits fictionnels théoriques.

Ces fictions théoriques ne sont pas de simples constructions arbitraires et dénuées de rapport à l'expérience concrète parce qu'elles sont fictionnelles. Butler prétend rendre compte d'une réalité concrète et non d'un empyrée ou d'une ontologie indépendants des accidents de l'expérience et de la matérialité. La fonction de sa philosophie n'est pas de donner ontologiquement l'être de son objet, mais d'en décrire le comportement effectif. À partir d'une description référentielle, l'enjeu est d'inférer la définition d'un processus. L'expérience de femme ou de lesbienne est non seulement celle de Butler, mais également de celles qui liront son ouvrage et pourront vérifier la capacité de sa philosophie à rendre compte de leur propre expérience singulière. La philosophie de Butler est un récit rendant compte d'une situation concrète effective, mais remise en forme dans la mesure où ce récit est censé décrire un processus permettant à tout individu d'atteindre à coup sûr l'objectif désiré, à condition de respecter la méthode indiquée. C'est une fiction, dans la mesure où le processus raconté est une opération mentale, sans être une fiction vraisemblable. Elle est désignée comme ayant un référent réel, dont l'expérience a une valeur générale. Il ne s'agit pas de quelque chose de fictif n'ayant pas de compte à rendre à l'expérience, mais simplement d'une fiction rendant compte d'une réalité. L'ensemble référentiel n'est pas pour autant vidé de matière de vérité, comme ce serait le cas dans un mensonge ou une autobiographie erronée, dans la mesure où ce qui est raconté n'est pas désigné comme ayant eu lieu exactement d'une manière historiciste.

\section{SE DIRE DE MANIERE PHILOSOPHIQUE}

Butler suggère dans plusieurs interviews que cette situation concrète effective est celle de sa propre vie. Si elle parle d'elle, c'est dans la mesure où son récit fictionnel théorique a pour fonction de lui permettre de se comprendre. Il faut entendre ceci à la fois au sens intensif de rendre compte de soi et au sens extensif de reconnaissance de sa propre expérience en la métaphorisant. C'est ce qui semble faire trace dans le texte, de sorte qu'il soit reconnaissable comme «parlant de son auteure », même si celle-ci n'apparaît pas explicitement; l'abstraction renvoyant les récits au statut de fiction vraisemblable et donc fictive se combine avec le référentiel.

Comme dans une autofiction, Butler se raconte, extrapole et considère cette extrapolation non seulement comme constitutive d'elle-même, mais comme révélant la vérité d'elle-même. Cette représentation qui ne correspond à aucune expérience précisément identifiable se 
retrouve dans chacune de ses expériences qui en sont l'expression. Ainsi, considérer une autoréférentialité de la philosophie de Butler n'est pas une falsification, mais au contraire « un dévoilement du moi appréhendé dans toutes ses dimensions, et notamment dans le rapport particulier, fictionnel, que ce moi entretient avec une vérité littérale, factuelle et événementielle $^{3} \gg$. Butler peut proposer un modèle philosophique excédant sa propre expérience tout en y renvoyant référentiellement. L'impersonnalité et la neutralité de l'écriture philosophique lui permettent de faire de la compréhension d'elle-même un modèle ayant une portée plus générale. Il s'agit donc d'un ouvrage philosophique établissant un compte rendu rétrospectif de la vie de l'auteur. En ce sens, il est possible de parler de philosophie autofictionnelle ${ }^{4}$.

Il peut être légitime de parler d'autofiction à propos d'un récit ayant une focalisation impersonnelle ou plus exactement indéterminée. Philippe Vilain considère la possibilité d'une autofiction anominale ou nominalement neutre, qui rende «appropriable le je par le plus grand nombre » :

Il s'agirait en quelque sorte de priver le je de lui-même, de l'autruifier [...], de l'orienter vers une esthétique moins intimiste qu'extimiste, une poétique de la distanciation, de manière à ce qu'il devienne à la fois, dans l'exercice de sa transparence et de son extensibilité, celui de personne et de tout le monde ${ }^{5}$.

Le je ne se cache pas pour autant, il se déplace et, se faisant, s'expose comme tel à qui sait le reconnaître. Il s'agit de se «généraliser et de puiser dans [s]a propre expérience des universaux, des invariants psychiques, de mettre à nu la logique propre d'une composante psychologique $^{6} \gg$.

Il semble que les constructions théoriques de Butler soient la compréhension plus que l'expression ou le compte rendu explicite de sa propre histoire. Le cas extrême pourrait être celui de la théorie philosophique de Butler dans La Vie psychique du pouvoir qui ne fait jamais référence à des situations concrètes mais qui pourtant propose un modèle permettant de les comprendre. Certains articles de Ces corps qui comptent ou du Pouvoir des mots sont par contre directement documentés, sans pour autant décrire exactement leur objet. Entre les deux, Trouble dans le genre se place à un certain niveau d'abstraction tout en explicitant

\footnotetext{
${ }^{3}$ Philippe Vilain, L'Autofiction en théorie, Paris, Les Éditions de la Transparence, 2009, p. 38.

${ }^{4} \mathrm{Au}$ sens où Dorrit Cohn parle d'autobiographie fictionnelle dans Le Propre de la fiction, Paris, Éditions du Seuil, 2001.

${ }^{5}$ Philippe Vilain, L'Autofiction en théorie, op. cit., p. 74.

${ }^{6}$ Ibid., p. 47.
} 
constamment le fait qu'il s'agisse de faire référence à des situations concrètes qui transparaissent sous la mise en forme théorique. Le rapport référentiel est condensé et déplacé, non littéral. Il est donné sur un mode qu'il est possible de qualifier de fantasme. Cela ne signifie pas qu'il n'est pas réel ou qu'il n'existe pas, mais qu'il est le produit d'une construction qui peut le rendre difficilement reconnaissable. Le récit renvoie à une expérience particulière mais prend une portée générale.

Butler met en forme son expérience selon les normes formelles de la philosophie généalogique, de sorte qu'il ne soit pas possible de discerner dans ses ouvrages la théorie de la référentialité. Comme la fiction pour l'autofiction, la philosophie est en un sens la condition transcendantale qui permet au lecteur de faire l'expérience de l'expérience de l'auteure. Il y a assimilation du réel à la fiction et, en plus, à la philosophie - fusion d'une théorie philosophique, d'une narration fictionnelle et d'un contenu autobiographique. En ce sens, l'œuvre de Butler est philosophique de la même manière que l'autofiction est fictive. Il serait sans doute possible de parler de «philosophication de soi », de la même manière que Philippe Gasparini parle de «fictionnalisation de $\operatorname{soi}^{7}$ ». L'auteure "philosophise » son existence à partir de sa déconstruction qui la met en récit. Si le contenu est alors majoritairement philosophique, l'hybridité n'en est pas moins également, et comme réciproquement, déséquilibrée du côté du sujet-auteur, en raison de son antériorité.

L'autoréférentialité semble doublement déplacée: une fois vers des situations qui paraissent emblématiques et permettent de dire quelque chose d'elle, tout en en généralisant la portée, et une seconde fois vers la théorie philosophique qui en fait un modèle. Ainsi, se met en place une ambiguïté, et à plus proprement parler sans doute, une tentative de rendre le moi énigmatique et difficilement lisible. La mise en fiction philosophique transforme le référent au point de le rendre suffisamment méconnaissable pour s'y référer, d'où le recours à l'impersonnalisation de la troisième personne qui, outre le fait qu'il renvoie au canon formel du traité philosophique, peut être considéré comme ayant une fonction dans l'économie autofictionnelle du texte. Dans sa pratique, l'autoréférentialité comme la fiction apparaissent dans leur acceptation la plus large. Pour reprendre les mots de Philippe Vilain, la philosophie dans l'autofiction théorique, comme la fiction dans l'autofiction, s'apparenterait davantage à une simple opération de traduction du réel et de récréation du référentiel : le vécu est transformé « en mettant en place les dispositifs opératoires de déplacement spatio-temporels, modifications, suppression ou supplémentation, condensation, synthétisation, traduction,

\footnotetext{
${ }^{7}$ Philippe Gasparini, Est-il je ? Roman autobiographique et autofiction, Paris, Éditions du Seuil, 2004, p. 11.
} 
décalage ${ }^{8} »$. Elle donne une série d'indices disséminés qui montrent qu'elle est concernée par ce qu'elle écrit, ou plus exactement que cela la concerne.

\section{LA FIGURE DE JUDITH BUTLER}

Il semble que la philosophie de Butler fasse signe vers sa propre histoire. Le texte ne prétend pas être une description historiciste de son auteure, mais son signe «bien plus complexe que le nom propre ${ }^{9} »$. Cette auteure à laquelle renvoient les ouvrages de Butler n'est pas Judith qui aurait été le témoin de sa propre vie. C'est Judith Butler comme figure constituée et mise en forme par l'auteur Butler. Il ne s'agit donc pas d'une biographie donnant la description d'un auteur empiriquement a priori déterminé. Judith Butler - pour dissocier cette figure qui est celle que perçoit le lecteur et qui lui donne ce nom d'un côté de la personnalité de l'individu, Judith qui répond à ce nom dans sa vie privée et de l'auteur Butler qui obéit à ce nom dans les taxinomies des bibliothèques - n'est donc pas qu'appréhendée au travers d'un corps textuel qui n'est pas vraiment le sien, et n'existe socialement que par la lecture de ce texte. Judith est inaccessible et Butler est une abstraction. Cela ne signifie pas que Judith n'existe pas et que Butler n'a pas d'intérêt. D'un côté, l'accessibilité à Judith est entièrement conditionnée par sa textualité. D’un autre côté, Butler renvoie à ce que Michel Foucault dénonce comme étant la « fonction auteur ${ }^{10} »$ qui a plus un rôle social dans la place culturelle de l'ouvrage que dans son économie propre.

L'auteur Butler apparaît ici d'une manière qui semble correspondre avec la conception d'Anders Buch-Jepsen pour qui, il «n'est donc pas une construction de l'origine du texte, mais une construction qui provient de l'interaction interprétative avec le texte ${ }^{11} »$. L'auteur présent n'est donc pas le référent réel, ce qui n'enlève rien à l'autoréférentialité du texte. Il est possible de considérer la référence à un auteur sans pour autant retomber dans ce que Michel Foucault dénonce comme étant la fonction auteur. La référence du nom sur la couverture d'un texte devient en fait, la figure de l'auteur construite dans le texte et non l'écrivant. Cette figure est celle qui se construit dans le texte et qui est le signe de Judith Butler, ni la description de la vie de Judith ni le simple nom propre vide Butler.

Il ne s'agit pas de dire que l'individu Judith n'a aucun rapport avec cette figure, mais qu'elle en est plus la construction qu'une description. La mémoire est détournée et Butler ne

\footnotetext{
${ }^{8}$ Philippe Vilain, L'Autofiction en théorie, op.cit., p. 22.

${ }^{9}$ Arnaud Schmitt, Je réel / je fictif : au-delà d'une confusion postmoderne, Toulouse, Presses universitaires du Mirail, 2010, p. 137.

${ }^{10}$ Michel Foucault, «Qu'est-ce qu'un auteur ? », dans Dits et écrits, 1954-1988, Paris, Gallimard, 1994, vol. 1.

${ }^{11}$ Anders Buch-Jepsen, cité par Arnaud Schmitt, Je réel / je fictif, op.cit., p. 141-143.
} 
témoigne pas de sa vie au sens courant du terme. Elle se retourne sur elle-même, mais pour donner sens à ce qu'elle a vécu - ou plus exactement pour se donner sens et apparaître dans le mouvement de sa propre existence de laquelle paradoxalement - et de manière cohérente avec sa théorie - elle est exclue. Butler considère qu'un sujet est parlé plus qu'il ne parle et, est agi plus qu'il n'agit. Plus exactement il n'existe comme sujet que dans la mesure où il se dit et apparaît selon certaines normes qu'il ne choisit pas mais dans lesquelles il se doit d'informer pour être reconnu comme tel. C'est à cette condition qu'il peut être conscient de lui-même comme individu agissant. Le sujet est exclu pour Butler de sa propre genèse, il porte la marque des normes dans lesquelles et par lesquelles il est reconnu comme tel. Il n'y a donc pas de je préalable au processus de subjectivation, et c'est précisément ce processus que décrit Butler. Si elle n'apparaît pas en tant que tel, c'est qu'à proprement parler, elle n'existe pas comme consciente d'elle-même par le mouvement qu'elle décrit. C'est l'écriture théorique de ce mouvement, le moment où elle reprend et recollecte sa propre histoire sociale et psychique, qui lui donne la conscience d'elle-même comme sujet.

Apparaît bien la difficulté d'établir une correspondance entre l'œuvre de Butler et l'impératif d'exactitude référentielle posé par Serge Doubrovsky pour définir une autofiction. Butler a une conception du sujet comme n'étant jamais donné. Il ne saurait donc être a priori référentiel : le référentiel s'informe et prend forme comme tel dans le mouvement même de l'écriture qui le reprend et le nomme plus qu'elle ne le transpose. Il faudrait, comme le suggère Philippe Vilain, parler de référentiel apocryphe ${ }^{12}$. Non seulement la mise en fiction transforme un référent au point de le rendre suffisamment méconnaissable pour s'y référer, mais plus encore elle le fait advenir comme tel. Il ne s'agit pas de dire qu'il n'y avait rien et que Judith n'existait pas avant de s'écrire ou qu'il ne s'est rien passé, mais que son histoire n'existait pas encore comme telle. Elle ne signifiait pas comme l'histoire de ce sujet qui advient conscient à lui-même, dans et par le mouvement de son écriture.

D'une manière similaire à Michel Foucault lorsqu'il considère qu'il n'écrit que pour se transformer, Butler ne semble écrire que pour se comprendre et parvenir à la conscience d'elle-même, de ses déterminations, comme de son agencivité. En un sens, Judith Butler n'existe comme telle qu'une fois son ouvrage achevé. Elle ne peut donc pas s'y présenter à la première personne. C'est donc peut-être paradoxalement compte tenu des normes du témoignage, que la non-utilisation d'un mode autodiégétique apparaît comme une garantie de crédibilité du texte comme autoréférentiel. La première personne est réputée conférer aux

\footnotetext{
${ }^{12}$ Philippe Vilain, L'Autofiction en théorie, op.cit., p. 33.
} 
énoncés les plus improbables une apparence de réalité et garantir l'historicité de ce qui est décrit dans un système social qui repose sur l'indispensable et pourtant illusoire croyance en l'objectivité des jugements émis par certaines premières personnes du singulier. C'est bien chez Butler, l'absence de l'usage de la première personne dans une œuvre décrivant des processus de subjectivation qui montre le lien consubstantiel avec sa vie.

Il reste dans la philosophie de Butler le procès de son existence. La forme même de l'écriture est le mouvement de la recherche de son existence. Butler, au sens strict, n'écrit pas - au sens de décrire, de donner à voir - son homosexualité et ses expériences. Cependant, elle écrit non seulement également à partir d'une philosophe qui se constitue de manière impersonnelle, mais également à partir d'un sans nom et sans image qui est un point subjectif évidé mais toujours présent. C'est à ce niveau que se joue la difficulté de la lecture de ses textes. Butler se rend abstraite pour renchérir sur le canon universitaire dans des ouvrages qui devraient par leur sujet l'excéder. Cette écriture complexe fait sens par rapport au caractère autofictionnel de sa philosophie. Résulte une forme difficilement lisible d'une écriture qui cherche un sujet grammatical qui ne parvient pas à se dire comme origine de son discours dans la mesure où il est socialement, culturellement et symboliquement invivable. Le sujet grammatical dans l'écriture de Butler fuit et cherche à se produire pour produire, justement, sa propre existence et sa propre légitimité. Cette fuite est la forme métaphorisée de celle de Judith se reproduisant et s'écrivant pour se créer une place sociale vivable, à la fois d'un côté comme femme et lesbienne, et en même temps comme philosophe.

Il y a une exigence de se dire qui est un devoir autant qu'une nécessité ontologique de dire. Si elle ne dit pas tout du détail de son expérience, elle la déplace pour dire tout ce que l'exigence et le devoir impliquaient qu'elle dît. Ce qui est dit n'a de valeur et de pouvoir qu'en ceci que l'expression est à la hauteur de l'enjeu - c'est-à-dire que s'ils ne sont à la hauteur à la fois de son expérience et de celle de ses lectrices. Il fallait le dire au lecteur, mais également à elle-même pour se comprendre et prendre par le mouvement de son écriture, conscience d'elle-même. Il fallait leur dire - au public et à elle-même - ce que la philosophie qu'elle a étudiée ne dit pas et qu'elle y a cherché : la possibilité de son existence. Ce qu'il lui fallait faire, c'est se donner une identité. C'est en écrivant qu'elle se crée, se forme et s'informe, se met en scène devant ce lecteur et devant elle-même, toujours troublée par le pouvoir des mots. C'est le processus de cette identité qui est en fait la sienne qui hante et imprime sa trace au texte philosophique de Butler : le trouble de son genre. Le livre n'est pas détachable de celle qui l'a écrit. 


\section{L'OUVERTURE D'UNE LECTURE POLYSEMIQUE}

Il est difficile d'appliquer à la philosophie de Butler la séparation radicale que propose Käte Hamburger pour définir une littérature par opposition à ce qu'elle définit comme étant le langage référentiel ${ }^{13}$. De la même manière que la lecture d'un roman pour Hamburger mais pour des raisons différentes, il peut sembler que la lecture d'un texte philosophique académique implique la conscience du fait que ce qui est écrit ou décrit n'est pas le compte rendu d'une expérience. La philosophie est différente de la réalité car elle implique la conscience de voir se déployer un monde conceptuel et métaphysique - au sens propre du terme. Elle ne semble exister que par le biais d'un acte discursif, contrairement à une description documentaire affirmée comme telle et certifiée par la présence de l'auteur présenté comme locuteur en tant que témoin.

Dans cette perspective, il faudrait dans le cas d'un ouvrage de philosophie, distinguer entre l'impersonnalité renvoyant au réel et la référence à l'expérience de l'auteur marquée par l'usage de la première personne. L'opposition peut sembler pouvoir être reprise pour distinguer une pratique philosophique académique formelle et orthodoxe de celle de l'essai. Elle n'apparaît pas comme opératoire à propos d'une philosophie ayant pour fonction de comprendre un réel concret dans lequel l'auteur est engagé et non de proposer une métaphysique par définition au-delà ou en deçà de toute expérience particulière. Il semble dur, en effet, de préserver dans l'œuvre de Butler l'intégrité du réel référentiel et de la fiction pour maintenir une frontière étanche.

Qualifier l'œuvre de Butler d'autofiction signifie que tout est arrivé mais que rien n'est arrivé comme elle le décrit, afin de mieux étudier le mode opératoire de l'identité de genre. Il est donc difficile de faire la part entre les différentes classifications génériques auxquelles peuvent renvoyer les textes de Butler. Sont entretenus trois modes génériques incompatibles de sorte que le texte est rendu énigmatique. Cette œuvre peut être lue et comprise dans des modalités différentes. Pour autant, elle n'est pas à la fois fictionnelle, autoréférentielle et philosophique. Elle est une composition inégalement pondérée. En l'occurrence, il s'agit d'une philosophie avec une dimension fictionnelle et autoréférentielle. Il faudrait nuancer cette caractérisation, qui peut sembler dépendre du point de vue du lecteur. D’un côté, le caractère philosophique de cette œuvre est assez évident. Cependant, pour la reconnaitre comme autoréférentielle, le lecteur doit connaitre son contexte d'écriture et des éléments de sa

\footnotetext{
${ }^{13}$ Käte Hamburger, Logiques des genres littéraires, Pierre Cadiot (trad.), Gérard Genette (préf.), Paris, Éditions du Seuil, 1986.
} 
biographie de l'auteur. Tout dépend du pacte de lecture actualisé par le lecteur. La même position est forcément polysémique si l'on prend le temps de la placer dans des contextes d'énonciation différents ${ }^{14}$.

Le texte ne présente pas pour autant cette alternance, mais il peut être lu selon ces différents points de vue. Les genres se superposent sans qu'on puisse les distinguer jusqu'à ce que ce complexe devienne un genre à part entière. Cependant, sans retomber dans l'idée d'une alternance au sein du texte entre ces différentes modalités, il est possible de considérer que la lecture se fait de manière différentielle en faisant jouer les pactes de lecture les uns avec les autres. Il semble que l'œuvre de Butler ne demande pas un pacte de lecture particulier. Le texte présenterait un nouveau genre indivisible et la lecture en ferait une composition de genres distincts. Ces deux idées ne sont pas nécessairement incompatibles. Dans la préface de Butler à Trouble dans le genre ${ }^{15}$, Butler présente un texte se voulant cohérent tout en instituant trois pactes de lecture emboîtés les uns dans les autres : à ceux de l'autobiographie, du pacte autobiographique et du pacte fictionnel, est ajouté un pacte philosophique. Ils s'imbriquent. Le premier est destiné à être actualisé par les militants dont la partie théoricienne peut, en même temps actualiser celui philosophique, et la partie mainstream celui $\mathrm{du}$ fictionnel. Le troisième est pris en charge par les philosophes, qui peuvent ou non actualiser également celui autofictionnel s'ils sont en même temps militants mais qui peuvent également le rejeter s'ils considèrent que la philosophie n'a pas à engager politiquement son auteur. Ils peuvent également ne pas prendre en charge celui fictionnel, s'ils considèrent que la philosophie est une affaire de théorie et non de fiction. Le pacte repose moins sur un contenu textuel que sur un engagement paratextuel.

Il est possible de reprendre l'idée de Philippe Gasparini selon laquelle l'existence même de l'autofiction, et donc de l'autofiction philosophique, repose sur une présupposition contextuelle fonction du groupe social du lecteur. Elle détermine la détermination générique du texte au niveau de sa lecture et interdit en fait une actualisation pleinement polysémique. Si le texte peut être hybride, la perception en est alternative : il passe d'un mode à l'autre ou du moins est toujours dominé par un mode qui impose sa logique aux autres. Apparaît alors un texte à choix multiples, qui se succèdent mais sans jamais se superposer. Il semble donc possible de récuser avec Philippe Gasparini ou Arnaud Schmitt l'idée d'une «hybridité

\footnotetext{
${ }_{14}^{14}$ Arnaud Schmitt, Je réel / je fictif, op.cit., p. 67.

15 Judith Butler, Trouble dans le genre : le féminisme et la subversion de l'identité [1990], Cynthia Kraus (trad.), Éric Fassin (préf.), Paris, La Découverte, 2005, p. 45-46.
} 
$\operatorname{cognitive~}^{16} \gg$ sans pour autant renoncer à l'idée de l'hybridité de l'œuvre qu'il dénonce et considère comme une illusion simplement due à une nouvelle figure de l'auteur. Ce serait simplement le résultat d'une fragilité et d'une complexification de ses engagements et d'un engagement de témoigner de la réintégration par le sujet de son histoire. Contre cette conception de l'impossibilité de la mixité, ce qui semble définir l'autofiction théorique, ce serait l'indécidabilité discursive - ce qui est différent du décalage qu'un texte produit nécessairement avec les normes de son genre a priori - et la nécessité d'une décision de lecture - ce qui ne signifie pas qu'elle soit consciente. L'instabilité n'est pas celle du texte mais bien celle de la figure de l'auteur identifié en fonction du pacte de lecture.

\section{CONCLUSION : UNE CONFESSION PHILOSOPHIQUE}

La pensée de Judith Butler peut être considérée comme une philosophie politique de l'identité donnant une boîte à outils susceptible d'être utilisée pour comprendre les mécanismes de subjectivation et de subordination d'une situation concrète quelconque. Elle se déploie cependant à propos de l'identité de genre, et en particulier de l'identité féministe, comme le précise le sous-titre de Trouble dans le genre. Elle semble en fait écrire une reconfiguration de son expérience de femme et de lesbienne selon les cadres et les normes du genre d'écriture qu'elle développe, c'est-à-dire d'une pratique philosophique qui est celle de la généalogie. L'individu Judith rend compte d'elle-même et nous apparaît sous cette forme. Elle s'adonne à ce qu'il est sans doute possible de considérer comme étant une confession philosophique. Reprenant la conception du «dernier Foucault», Butler définit dans Le Récit de soi la confession comme l'acte discursif par lequel le sujet se rend public, se donne en mots en reprenant et en assumant les diverses influences qui agissent sur son écriture ${ }^{17}$. Elle se présente comme philosophe, ou plus exactement sur une scène qui est celle de la philosophie et où elle veut être reconnue. Elle n'en met pas moins à jour la vérité et la réalité concrète de son expérience sous la forme de récits y renvoyant. Butler construit un compte rendu philosophique de sa propre vie - contre d'un côté une position historiciste considérant que ce qui est décrit l'est exactement et contre une position métaphysicienne considérant qu'il est possible de conceptualiser la vérité du monde sans le décrire. Butler échappe à l'illusion, qu'elle dénonce d'ailleurs, de l'édiction d'une vérité objective sur soi.

L'œuvre de Butler invite à reconsidérer la notion même de vérité référentielle « dont il ne faudrait pas réduire la définition à une factualité et à une événementialité trop stricte, mais en

\footnotetext{
${ }_{16}^{16}$ Arnaud Schmitt, Je réel / je fictif, op.cit., p. 45-72.

${ }^{17}$ Judith Butler, Le Récit de soi [2005], Bruno Ambroise \& Valérie Aucouturier (trad.), Paris, Presses universitaires de France, 2007, p. 113-116.
} 
élargir le sens au contraire à une distinction entre un référentiel réel et un référentiel fictif, entre un référentiel objectif et un référentiel subjectif ${ }^{18} »$.

Elle refuse la position d'un sujet transhistorique rendant compte d'une expérience de pensée. Ce faisant, elle sort de l'épistémè philosophique classique dominée par la forme du traité impersonnel pour s'inscrire dans une autre attitude philosophique. Pour reprendre les termes de Michel Foucault qu'elle cite dans Le récit de soi, Butler produit une place pour l'intrigue d'un «je» au sein du régime discursif de la philosophie ${ }^{19}$. Elle propose une nouvelle forme qu'il est possible de désigner sous le terme d'autofiction théorique. Il ne s'agit de présager ni des intentions de l'auteur, ni de la réception du texte, mais de reconnaître dans celui-ci une compréhension de l'expérience du premier et des traces de cette expérience susceptibles d'être reconnues lors de la seconde. Peu importe que le lecteur doute de la véridicité des faits racontés et s'interroge sur la nature du pacte adopté, puisque le texte se présente comme philosophique et militant. Comme pour toute autofiction, libre à lui de le lire comme une autobiographie ou comme un traité. Si alors son attente est déçue, il sera responsable de cette déception.

Par cette forme, Judith Butler gagne la légitimité philosophique académique du côté de l'abstraction théorique, la légitimation politiquement progressiste du côté de sa pratique du récit de déconstruction et la légitimité militante du côté de la référence à son expérience de subalterne. Elle ne s'abrite pas derrière la forme fictionnelle ou la forme philosophique pour échapper à la responsabilité sociale et culturelle qui est celle d'un auteur dont l'œuvre a été réappropriée politiquement, pas plus qu'elle ne se cache derrière la forme militante pour perdre sa rigueur conceptuelle ou pour tomber dans la facilité d'une position unilatérale. Elle élargit au contraire et rend instable le contrat de lecture en troublant les positions afin de représenter et de mettre en scène leur porosité dans le déploiement non seulement de son écriture, mais essentiellement à ce niveau de sa lecture. C'est de cette forme qu'elle tient la force et la complexité de son engagement, sans en renier aucune des composantes. Cette force qui fait porter sa pensée ne vient pas de la précision sociologique d'une observation ou de l'affirmation narcissique d'une parole dont elle seule pourrait porter la vérité sous prétexte qu'elle a fait l'expérience de ce dont elle parle. Elle vient au contraire des écarts dans lesquels Michel Foucault voyait le lieu et le mouvement de la constitution d'une vérité qu'il

\footnotetext{
${ }^{18}$ Philippe Vilain, L'Autofiction en théorie, op.cit., p. 33.

${ }^{19}$ Judith Butler, Le Récit de soi, op.cit., p. 114.
} 
considérait comme multiple et multiforme ${ }^{20}$. Dans cette forme, bien plus que dans l'autobiographie ou toutes les formes d'historicisme, est peut-être le «courage de la vérité », la parrhésia qui, lorsqu'elle s'adresse aux hommes, est celle de la philosophie qui, plutôt qu'à la métaphysique et à l'empyrée, s'attache à la vie - ou plus exactement s'attache à être vie.

\footnotetext{
${ }^{20}$ Michel Foucault, Le Courage de la vérité, Le Gouvernement de soi et des autres II : cours au Collège de France 1983-1984, Paris, Éditions du Seuil / Gallimard, « Hautes études », 2009.
} 